\title{
Just Noticeable Differences in the Length of Golf Irons ${ }^{+}$
}

\author{
Josh P. Sumner *, Jonathan R. Roberts and Aimée C. Mears \\ Sports Technology Institute, Wolfson School of Mechanical, Electrical and Manufacturing Engineering, \\ Loughborough University, Loughborough LE11 3QF, UK; J.R.Roberts@lboro.ac.uk (J.R.R.); \\ A.C.Mears@lboro.ac.uk (A.C.M.) \\ * Correspondence: J.Sumner@lboro.ac.uk; Tel.: +44-150-956-4811 \\ + Presented at the 13th conference of the International Sports Engineering Association, Online, \\ 22-26 June 2020.
}

Published: 15 June 2020

\begin{abstract}
Current custom fitting guidelines for golf clubs suggest the smallest change in club length from a standard length iron should be $6 \mathrm{~mm}\left(1 / 4^{\prime \prime}\right)$. However, no previous research suggests why this length change is used. This study aims to identify the minimum noticeable difference in the length of 7-iron clubs using just noticeable difference analysis techniques. Fifty golfers of varying ability were asked to compare a standard length 7-iron to test 7-irons of varying lengths, regarding changes in the perception of club length and body position at address. Irrespective of golfing ability, golfers could perceive a just noticeable difference of $13 \mathrm{~mm}\left(1 / 2^{\prime \prime}\right)$ from the standard length club. However, when asked if changes in body position were present, category 1 golfers noticed differences with a $6 \mathrm{~mm}$ change in club length and category 2 golfers noticed differences with a 13 $\mathrm{mm}$ change. No changes in body position were perceived by highly handicapped golfers.
\end{abstract}

Keywords: golf equipment; custom fitting; perception; golfer

\section{Introduction}

Golfers commonly try to improve their game beyond what is possible from training alone and may seek clubs that are recommended to them during custom golf club fitting sessions based on their anthropometrics and swing technique. A wide variety of clubs are available during a fitting due to manufacturers offering numerous club heads, shafts and grips with differing properties, which makes determining the most suitable club for an individual golfer a challenging task.

The club length fitting method commonly uses guidelines based on a combination of a golfer's height and wrist/knuckle to floor distance to statically determine an appropriate club length. Current club fitting guidelines suggest the minimum change in iron club length from a standard club length should be $6 \mathrm{~mm}\left(1 / 4^{\prime \prime}\right)$ [1]. Following the static fitting, golfers will perform several shots to confirm if the club is the correct length, generally based on a combination of intrinsic feedback from the golfer and extrinsic feedback from the fitter or a launch monitor, providing measurements of club and ball at impact. The effect of a $6 \mathrm{~mm}$ change in club length has on a golfer is currently unresearched, but PING suggest for each $13 \mathrm{~mm}$ increase in 7-iron club length, increases should be observed in ball speed $(+1 \mathrm{mph})$, clubhead speed $(+1.5 \mathrm{mph})$, launch angle $\left(+0.5^{\circ}\right)$, spin rate $(+50 \mathrm{rpm})$ and carry distance $(+1.4 \mathrm{~m})$ [2]. Previous research has shown ball velocity slightly increases with a $\approx 13 \mathrm{~mm}$ driver club length increase, however, contradicting research has shown that increases of $51 \mathrm{~mm}$ in driver length do not significantly affect clubhead and ball parameters, suggesting even smaller changes of $6 \mathrm{~mm}$ may have little effect on performance [3,4].

Club length has been shown to affect both a golfer's ball velocity and swing technique at the address position (i.e., the start of the swing) and during the swing [5-7]. However, most of the 
previous research has primarily compared differences between various types of clubs that have much greater club length differences than the manipulations used in club fitting. Therefore, further research needs to be conducted to understand how small length changes used in club fitting affect a golfer's biomechanics.

As well as a lack of research analysing how the small club length changes used in custom fitting affect a golfer, it is unknown if changes of that size are perceivable. It is believed that a perceived change in the equipment will elicit a change in the golfer's confidence when using the club, leading to a change in shot performance [8]. By understanding the club length changes that are perceivable, we can begin to comprehend which club length changes may affect a golf swing. Just noticeable difference (JND) analyses utilise qualitative methods to assess the smallest noticeable change in a given stimulus; these analysis techniques are widely used in the food and beverage industry [9], but have previously been used to assess the threshold of sensitivity to changes in sporting equipment [10]. Webber's law indicates that the JND of a stimulus is proportional to its intensity, indicating a measured JND in golf club length can be applied across different club types [11].

The current study aims to analyse the JND in 7-iron club length based on perceived changes to club length and a golfer's body position at address (i.e., posture). It is hypothesised that a change of $6 \mathrm{~mm}$ in club length will be detectable using JND analysis techniques.

\section{Materials and Methods}

\subsection{Equipment}

Nine different 7-iron lengths were used during data collection. A standard length (STD) 7-iron club (94.0 cm [37"]), and 7-irons with varying length changes in both directions $( \pm 13,6,3$ and $2 \mathrm{~mm}$ $\left[1 / 2^{\prime \prime}, 1 / 4^{\prime \prime}, 1 / 8^{\prime \prime}\right.$ and $\left.1 / 16^{\prime \prime}\right]$ ) were used [1]. These increments were selected to represent the two smallest changes present in club fitting, along with two smaller increments. The clubs used in the present study were built from an Acer XV Professional 7-iron clubhead (Mass $=270 \mathrm{~g}$, Loft $=33^{\circ}$, Lie $=62.5^{\circ}$ ), regular stiffness Dynamic Gold shafts (Mass $[S T D]=109.1 \mathrm{~g}[ \pm 1.3 \mathrm{~g} / \mathrm{cm}$ with change in club length]) and Golf Pride Dual Durometer grips (Mass $=50 \mathrm{~g}$ ). To ensure accurate length measurement, the clubhead was 3D scanned (ATOS Core, GOM GmbH, Braunschweig, Germany) to measure the distance from the intersect point of the shaft plane and sole plane to the base of the hosel $(25.4 \mathrm{~mm})$, allowing the shafts to be trimmed to the required lengths. Each shaft was fitted with two $8 \times 8 \mathrm{~mm}$ cylindrical neodymium magnets ( $3 \times g$ each), one glued flush to the tip of the shaft and the other protruding from the end of the shaft, allowing for the quick attachment and detachment of the clubhead while securing it sufficiently for the testing protocol.

\subsection{JND Protocol (Method of Constant Stimuli)}

The protocol followed the method of constant stimuli, where a series of test conditions were compared to a reference condition, the central point of the series [9]. Fifty mixed ability golfers (48 males, two females; Height: $178 \pm 10 \mathrm{~cm}$; Wrist to floor: $87.9 \pm 4.9 \mathrm{~cm}$ ) were recruited. Participants were grouped into self-reported handicap categories, defined by the Council of National Golf Unions Handicapping System, with category 4, 5 and 6 combined (category 4+) [12]. Category 1 golfers had a handicap of 5.4 or less $(n=8)$; category 2 with handicaps of 5.5-12.4 $(n=10)$, category 3 with handicaps of $12.5-20.4(n=18)$ and category $4+$ with handicaps of 20.5 or higher $(n=14)$. Full ethical approval was granted by Loughborough University and all participants gave full informed consent.

Each participant attended one session and the session was split into two parts. Before each part of the session, the participants performed a series of familiarisation trials, comparing randomly selected clubs. Firstly, participants were asked to adopt an address position with the STD club to assess its length, then repeat with an alternative length club. They were asked to respond "yes" or "no" to the question: "Are the clubs the same length?" This was repeated with all eight of the test clubs in a randomized order. Following completion of the final pair comparison, the participant was asked to explain the technique they used to determine a change in length. Secondly, the test was repeated, however this time, the participant was told to think about how their posture changed 
between clubs and were asked: "Is your posture the same with both of the clubs?" If a change in posture was present, the participant was asked to explain what had changed in more detail.

The testing procedure was explained to the participants and they were informed that some clubs were the same length, some were slightly different lengths, and some had greater differences. This deception was necessary to ensure participants were only reporting a difference when one was perceived. Following the end of the session, the participants were debriefed, and the deception was explained. The participants only held the club in address position and were refrained from performing any swings, half-swings or 'waggles', negating any perceivable differences in the swing weight or moment of inertia due to the length adjustment.

\subsection{Data Analysis}

The JND is suggested to be the smallest physical difference that can be perceivably identified more than $50 \%$ of the time [9]. As the participants were asked to respond "yes" or "no" to whether they perceived the club length or their posture to be the same between pairs of clubs, a negative response indicated a difference was perceived. When the proportion of negative responses exceeded $50 \%$, a JND was present. To correct for the probability of a correct response by chance, a correction proposed by Gacula \& Kubala [13] was applied:

$$
\mathrm{P}=2 \mathrm{P}^{*}-1,0 \leq \mathrm{P} \leq 1.0
$$

where $\mathrm{p}^{*}$ is the actual proportion of negative responses and $\mathrm{P}$ is the corrected proportion. The corrected proportions of matching plus and minus length changes were combined, allowing for an analysis of the change in club length, irrespective of direction. The data were firstly analysed as a global population, then participants were grouped to determine how golfing ability affected golfer perceptions of changes in club length.

Z-Scores were calculated, indicating the number of standard errors (SE) a value is from the $50 \%$ JND threshold;

$$
S E=\sqrt{1 / n}
$$

where $n$ is the number of participants. Large positive Z-scores $(\geq 1.96)$ indicate a noticeable difference with a $95 \%$ confidence interval $(\mathrm{CI})$ and large negative Z-scores $(\leq-1.96)$ indicates an imperceptible difference $(95 \% \mathrm{CI})$.

Weber's law indicates that the JND of a physical stimulus is proportional to the original stimulus intensity. The Weber fraction is therefore the constant of Weber's Law, indicating the sensory system's ability to detect changes in stimuli and was calculated using the following equation:

$$
\Delta \mathrm{I} / \mathrm{I}=\mathrm{k}
$$

where $\Delta \mathrm{I}$ is the JND from the starting physical stimulus, I [9].

\section{Results}

\subsection{JND of Change in Club Length}

The percentage of negative responses increased as the change in club length increased; however, only $13 \mathrm{~mm}$ was perceived to be a JND, with $68 \%(\mathrm{Z}=3.00)$ of the responses perceiving a difference (Figure 1a). The Weber fraction for perceiving a change in club length was $13.83 \times 10^{-3}$. These findings were representative across all handicaps, with each category perceiving a JND of $13 \mathrm{~mm}$ change in club length. While Z-scores, except 'category 1', were less than 1.96, all expressed a $>80 \% \mathrm{CI}$, suggesting that with an increased number of participants, a 95\% CI could be found (See Table 1).

When golfers were asked how they determined a change in length, 38\% of all participants visually observed a change in the distance of the clubhead from the centre of their stance, $24 \%$ perceived a change in trunk flexion and $24 \%$ reported a change in their grip position. Others noted a change in the general feel of the club, effective lie angle of the club, shoulder flexion and balance. 


\subsection{JND of Change in Posture}

The mean percentage of responses indicating a perceivable difference in posture due to a change in club length increased proportionally with change in club length. However, all length changes represented an imperceptible difference $(Z<-1.96)$, suggesting the JND is greater than $13 \mathrm{~mm}$. When explored based on a golfer's ability, highly skilled golfers were able to perceive changes in posture better than lower skilled golfers (Figure 1b). Category 1 golfers expressed a JND in posture when the club length changed by $6 \mathrm{~mm}(Z=2.00)$. Category 2 golfers had a response rate of $50 \%$ for the $13 \mathrm{~mm}$ length change, suggesting that there may or may not have been a perceivable difference in their posture (Table 1). The Weber fraction for perceiving changes in posture due to a change in club length was $6.383 \times 10^{-3}$ for category 1 handicap golfers and $13.83 \times 10^{-3}$ for category 2 handicap golfers.

When asked what aspect of the posture changed between clubs, across all golfers, the most common responses were a change in trunk flexion ( $82 \%$ ) and knee flexion $(46 \%)$. A selection of golfers adjusted their setup to counteract to the change in club length by either altering the stance-ball distance (18\%) or gripping the club differently to negate the change in club length $(12 \%)$. Other golfers mentioned a change in shoulder flexion, balance, shoulder elevation and the curvature of the spine.

(a)
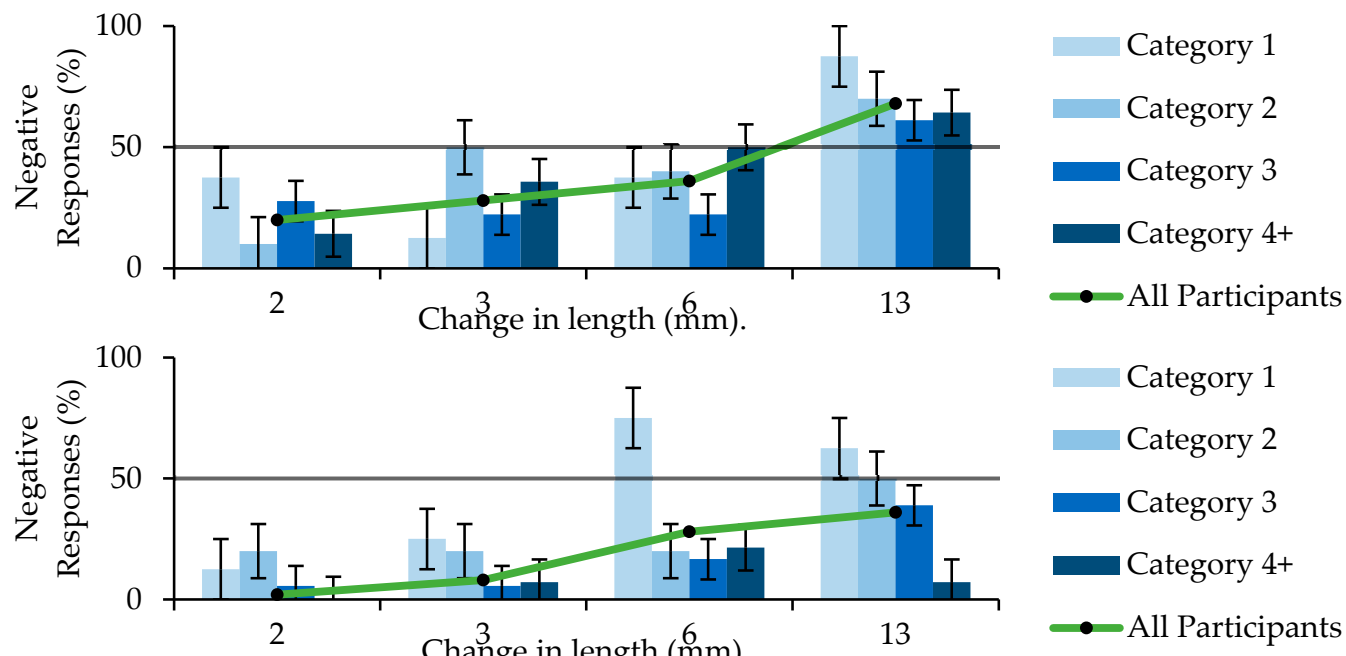

Figure 1. Mean \pm SE percentage of negative responses across all participants and each handicap category perceiving a difference in (a) the club length and (b) the posture at the address position across varying length changes. $\mathrm{A}>50 \%$ difference (solid black line) indicated a noticeable difference.

Table 1. Z-Score measurements for noticeable difference in club length and posture across varying length changes (2-13 $\mathrm{mm}$ ) for each handicap category.

\begin{tabular}{ccccccccc}
\hline & \multicolumn{8}{c}{ Noticeable Differences (Z-Scores) } \\
\hline & \multicolumn{7}{c}{ Club Length } & \multicolumn{5}{c}{ Posture } \\
\hline Handicap Category & $\mathbf{2} \mathbf{~ m m}$ & $\mathbf{3} \mathbf{~ m m}$ & $\mathbf{6} \mathbf{~ m m}$ & $\mathbf{1 3} \mathbf{~} \mathbf{m}$ & $\mathbf{2 ~} \mathbf{~ m}$ & $\mathbf{3} \mathbf{~ m m}$ & $\mathbf{6} \mathbf{~ m m}$ & $\mathbf{1 3} \mathbf{~ m m}$ \\
\hline $\mathbf{1}$ & -1.00 & -3.00 & -1.00 & 3.00 & -3.00 & -2.00 & 2.00 & 1.00 \\
$\mathbf{2}$ & -3.58 & 0.00 & -0.89 & 1.79 & -2.68 & -2.68 & -2.68 & 0.00 \\
$\mathbf{3}$ & -2.67 & -3.33 & -3.33 & 1.33 & -5.33 & -5.33 & -4.00 & -1.33 \\
$\mathbf{4 +}$ & -3.78 & -1.51 & 0.00 & 1.51 & -5.29 & -4.54 & -3.02 & -4.54 \\
All Participants & -6.00 & -4.40 & -2.80 & 3.60 & -9.60 & -8.40 & -4.40 & -2.80 \\
\hline
\end{tabular}

\section{Discussion}

The present research has identified two key findings. First, when asked to determine if a change in club length was present, golfers were able to perceive a JND of $13 \mathrm{~mm}$, irrespective of golfing ability. Secondly, higher skilled golfers were able to notice changes in posture at address when club length was changed by $6 \mathrm{~mm}$, compared to low-ability golfers who were unable to perceive changes in posture across any of the conditions used. 
An incorrect posture at the address position could detrimentally effect a golfer's swing [14]. Therefore, it could be assumed that if a golfer is able to perceive a change in posture, a change in swing biomechanics could follow. The present study suggests that when addressing the ball, category 1 handicapped players were able to perceive a change in posture at a club length change of $6 \mathrm{~mm}$, which could influence their swing. However, category 3 and $4+$ golfers could not perceive a $13 \mathrm{~mm}$ change and, therefore, it is assumed that manipulation of their 7-iron length by that amount would not influence their swing. Depending on golfer height and wrist to floor measurement, golf club fitters currently adjust club length by a minimum of $6 \mathrm{~mm}$. The present study suggests that this minimum length adjustment may only be appropriate for category 1 golfers, while a minimum of a $13 \mathrm{~mm}$ adjustment may be more appropriate for category 2 golfers.

A golfer will use several different clubs during a game of golf and each club varies in length. The long irons (2-, 3- \& 4-irons) and woods are longer than a 7-iron, and the short irons (8- \& 9-iron) and wedges are shorter again. Weber's law suggests that with an increase in stimulus intensity, JND will increase proportionately [11]. As the ratio of the JND and intensity, Weber's index suggests that, for a category 1 golfer, the JND of club length to incite a perceived change in posture from a STD driver $(115.6 \mathrm{~cm})$ would be $7 \mathrm{~mm}$ and $16 \mathrm{~mm}$ for category 2 golfers. However, further work would have to be conducted to confirm these findings.

While golfers of all abilities were able to perceive a change in club length of $13 \mathrm{~mm}$, the likelihood of a golfer directly analysing a change in club length is low; therefore, perceptions of a change in posture are a more appropriate measurement. However, during the testing, golfers were asked to apply selective attention to their posture, which could increase sensitivity to changes [15]. Predictive coding models have suggested that with increased attention, the weighting of sensory signals that are both sensory salient and behaviourally relevant are improved [15,16]. Previous research has shown that, the primary and secondary most discernible properties of feel when driver club length was manipulated were the feeling of shaft stiffness and sound at impact [17]. This indicates that when selective attention is not applied to the posture of the golfer, attention may shift and the perceivable differences between the clubs may decrease.

The maximum swing weight difference between the STD and test clubs in the present study was four points. It has been shown that most players can only perceive swing weight changes of five points or more, suggesting that mass changes present in the study would not have interfered with the participant's perception of length change [18].

Further research should focus on analysing if the perceivable differences measured in the present study cause a measurable change in the posture of a golfer at both the address position and throughout the swing using biomechanical analysis. In the address position, when comparing a 7-, 5, 3-iron and a driver, multiple variables of the setup have been reported to change, such as stance width, trunk flexion and right-side bending [5]. During the swing, when comparing a driver to a 7iron, peak trunk flexion and left side bending decreased, as did right side bending velocity, which has implications for both performance and injury risk [6,19]. Both previous studies looked at interclub comparisons with at least a $24 \mathrm{~mm}$ difference between club lengths. However, further research needs to investigate how a just noticeable change in club length effects the posture of a golfer, allowing golf club fitters to understand how the length adjustments made during a fitting session can influence the movement and performance of a golfer.

\section{Conclusions}

Golfers followed the method of constant stimuli to determine the JND of a change in club length from a standard length 7-iron. All golfers expressed a JND of $13 \mathrm{~mm}$ from the standard. However, when addressing the ball, category 1 golfers were able to observe a just noticeable change in posture when club length was manipulated by just $6 \mathrm{~mm}$. Category 2 golfers only recognised a just noticeable change in posture at a $13 \mathrm{~mm}$ change in club length and higher handicapped golfers were not able to notice a change in posture for any of the conditions tested.

Due to increased perceptual sensitivity to postural changes, this study confirms the current minimal club length change used by club fitters be appropriate for category 1 golfers; however, it 
could be suggested that weaker golfers should be fitted to looser guidelines for club length than currently suggested. These findings should be confirmed through a biomechanical analysis to investigate if these JNDs cause a change in swing biomechanics and shot outcome.

Funding: This research received no external funding

Conflicts of Interest: Authors declare no conflict of interest.

\section{References}

1. TaylorMade. Custom-Fitting Manual, 2013. Available online: https://www.yumpu.com/en/document/ read/11578723/custom-fitting-manual-taylor-made-golf (accessed on 16 July 2019).

2. PING. PING Fitting Manual, 2017. Available online: https://issuu.com/pingeurope/docs/ping_fitting_ manual_2017 (accessed on 16 July 2019).

3. Lacy, T.E.; Yu, J.; Axe, J.; Luczak, T. The effect of driver mass and shaft length on initial golf ball launch conditions: A designed experimental study. Conf. Int. Sport. Eng. Assoc. 2012, 34, 379-384, doi:10.1016/j.proeng.2012.04.065.

4. Kenny, I.C.; Wallace, E.S.; Otto, S.R. Influence of shaft length on golf driving performance. Sport. Biomech. 2008, 7, 322-332, doi:10.1080/14763140802233249.

5. Sung, R.J. Changes of setup variables by the change of golf club length. Korean J. Sport Biomech. 2005, 15, 95-104, doi:10.5103/KJSB.2005.15.3.095.

6. Lindsay, D.M.; Horton, J.F.; Paley, R.D. Trunk motion of male professional golfers using two different golf clubs. J. Appl. Biomech. 2002, 18, 366-373, doi:10.1123/jab.18.4.366.

7. Joyce, C.; Burnett, A.; Cochrane, J.; Ball, K. Three-dimensional trunk kinematics in golf: Between-club differences and relationships to clubhead speed. Sport. Biomech. 2013, 12, 108-120, doi:10.1080/14763141.2012.728244.

8. Roberts, J.; Jones, R.; Harwood, C.; Mitchell, S.; Rothberg, S. Human perceptions of sports equipment under playing conditions. J. Sports Sci. 2001, 19, 485-497, doi:10.1080/026404101750238944.

9. Lawless, H.T.; Heymann, H. Sensory Evaluation of Food: Principles and Practices; Springer: Berlin, Germany, 2010.

10. Bower, R.; Cross, R. Player sensitivity to changes in string tension in a tennis racket. J. Sci. Med. Sport. 2003, 6, 120-131, doi:10.1016/S1440-2440(03)80015-4.

11. Fechner, G.T.; Davis, H.H.; Boring, E.G. Elements of Psychophysics; Holt, Rinehart and Winston: New York, NY, USA, 1966; Volume 1.

12. CONGU. CONGU Unified Handicapping System, 2019. Available online: https://www.congu.co.uk/ (accessed on 16 July 2019).

13. Gacula, M.C.; Kubala, J.J. Weighting coefficients for the estimation of sensory threshold. Chem. Senses 1978, 3, 105-121, doi:10.1093/chemse/3.1.105.

14. Smith, A.; Roberts, J.; Wallace, E.; Forrester, S. Professional golf coaches' perceptions of the key technical parameters in the golf swing. Procedia Eng. 2012, 34, 224-229, doi:10.1016/J.PROENG.2012.04.039.

15. Thielen, H.; Gillebert, C.R. Sensory sensitivity: Should we consider attention in addition to prediction? Cogn. Neurosci. 2019, 10, 158-160, doi:10.1080/17588928.2019.1593125.

16. Rao, R.P.N. Bayesian inference and attentional modulation in the visual cortex. Neuroreport 2005, 16, 18431848, doi:10.1097/01.wnr.0000183900.92901.fc.

17. Iwatsubo, T.; Nakajima, D. Evaluation of long and short shafts of golf club by real swing. In The Engineering of Sport 6; Springer: New York, NY, USA, 2006; pp. 347-352.

18. Harper, T.E.; Roberts, J.R.; Jones, R. Driver swingweighting: A worthwhile process? Proc. Inst. Mech. Eng. Part B J. Eng. Manuf. 2005, 219, 385-393, doi:10.1243/095440505X32247.

19. Sato, K.; Kenny, I.; Dale, B. Current golf performance literature and application to training. J. Trainology. 2013, 2, 23-32, doi:10.17338/trainology.2.2_23. 